\title{
ESTUDIOS DE PROCEDENCIA (SUBMAREAL/INTERMAREAL) DE MEJILLONES (MYTILUS PLATENSIS) EN LA LOCALIDAD ARQUEOLÓGICA FARO SAN MATÍAS (GOLFO SAN MATÍAS, RIIO NEGRO, ARGENTINA)
}

PAMELA G. STEFFAN ${ }^{a}$, ENRIQUE MORSAN ${ }^{b} \&$ CRISTIAN M. FAVIER DUBOIS ${ }^{a}$

\begin{abstract}
RESUMEN
Los mitílidos son uno de los recursos marinos más utilizados en el pasado y muy frecuentes de hallar en el registro arqueomalacológico de zonas costeras. En la costa atlántica norpatagónica el mejillón común Mytilus platensis está presente tanto como vestigio arqueológico de su consumo pasado como en poblaciones vivientes en aguas someras. El objetivo de este trabajo es explorar una metodología tendiente a reconocer zonas de colecta de este recurso malacológico en el pasado e inferir modos de aprovisionamiento del mismo. El enfoque metodológico se respalda en el análisis conjunto de las relaciones alométricas de valvas enteras provenientes de diez concheros de la localidad arqueológica Faro San Matías (Río Negro) y las actuales colectadas en zonas litorales del intermareal y submareal del golfo San Matías. Las valvas de la zona submareal registran un patrón de crecimiento alométrico diferente respecto a las del intermareal y, en consecuencia, es posible discriminar la zona de procedencia de los especímenes. Así, el patrón observado en las valvas actuales $(n=216)$ permite inferir el sector de recolección en las arqueológicas $(n=932)$, a lo que pueden sumarse otros criterios biológicos. Como resultado de este estudio, se ha observado que las valvas de tres sondeos de la localidad Faro San Matías sugieren mezcla de zonas de colecta, intermareal y submareal, mientras que las de otros cuatro sondeos analizados estarían indicando una zona de procedencia submareal. Finalmente, se discuten estos datos a partir de test estadísticos para establecer valores discriminantes entre las valvas y aproximarnos a las estrategias de aprovisionamiento utilizadas durante el Holoceno tardío en este litoral.
\end{abstract}

PALABRAS CLAVE: arqueomalacología, Mytilus platensis, zona litoral, estrategias de aprovisionamiento, Holoceno tardío.

a INCUAPA- CONICET, Facultad de Ciencias Sociales, Universidad Nacional del Centro de la Provincia de Buenos Aires. Del Valle 5737. B7400JWI. Olavarría, Argentina. \psteffan@soc.unicen.edu.ar

b Centro de Investigación Aplicada y Transferencia Tecnológica en Recursos Marinos "Almirante Storni" (CIMAS) /Prov. de Río Negro - Universidad Nacional del Comahue - CONICET, San Antonio Oeste, Río Negro. 


\title{
PROVENANCE STUDIES (SUBTIDAL / INTERTIDAL) OF MUSSELS (MYTILUS PLATENSIS) IN FARO SAN MATÍAS ARCHAEOLOGICAL LOCALITY (SAN MATÍAS GULF, RÍO NEGRO, ARGENTINA)
}

\begin{abstract}
Mussels are one of the most used marine resources in the past and very frequent in the archaeomalacological record of coastal areas. On the North Patagonian Atlantic coast, the common mussel, Mytilus platensis, is recorded both as an archaeological evidence of its past consumption and as living populations in shallow waters. The objective of this work is to explore a methodology to recognize harvesting areas of this malacological resource in the past and infer modes of exploitation. The methodological approach is supported by the analysis of the allometric relationships of whole valves from ten shell-middens from the archaeological locality Faro San Matías (Río Negro) in comparison with those collected currently in intertidal and subtidal littoral zones of the San Matías Gulf. Shells of the subtidal zone present a different allometric growth pattern with respect to the intertidal ones and, consequently, it is possible to discriminate the zone of provenance of the specimens. Thus, the pattern observed in the current valves $(n=216)$ allows us to infer the collection sector in the archaeological ones $(n=932)$, to which other biological criteria can be added. As a result of this study, it has been observed that the valves of three shell-middens of the locality Faro San Matías suggest mixing of collection areas, intertidal and subtidal, while those of another four shell-middens would indicate a subtidal provenance. Finally, these data are discussed with statistical tests to establish discriminant values among the valves and to approach the procurement strategies used during the Late Holocene in this coast.
\end{abstract}

KEY WORDS: Archaeomalacology, Mytilus platensis, littoral zone, procurement strategies, Late Holocene.

\section{INTRODUCCIÓN}

El estudio de los moluscos en Arqueología es referido a la Arqueomalacología (Claassen, 1998; Bar-Yosef Mayer, 2005; Cakirlar, 2011; Hammond \& Zubimendi, 2015), a partir de la cual se aborda una diversidad de cuestiones antropológicas y paleoambientales (Claassen, 1998). En la costa patagónica argentina, las identificaciones taxonómicas de moluscos provienen de terrazas marinas del Cuaternario (Pastorino, 2000; Aguirre et al. 2009; entre otros). La mayoría de los análisis arqueomalacológicos provenientes de sitios costeros incluyen la composición y preservación de los concheros (Orquera \& Piana, 2001; Hammond, 2014, 2015), contribución dietaria (Orquera y Piana 2001; Santiago et al. 2014), morfometría (Lobbia, 2016) y estacionalidad (Lobbia, 2012; Steffan \& Morsan, 2015; Favier Dubois et al. 2015), desde una perspectiva local o regional (Zubimendi et al. 2005; Hammond et al. 2015). Los enfoques metodológicos para reconocer zonas de colecta o procedencia de recursos malacológicos han estado usualmente restringidos al estudio de las características ecológicas generales de los taxones. Por tal motivo el objetivo de este trabajo es aportar otros criterios al estudio del aprovisionamiento de moluscos, específicamente de Mytilus platensis (mejillones), a partir de un caso de estudio en la localidad arqueológica Faro San Matías (FSM), ubicada en el litoral atlántico del norte de Patagonia (golfo San Matías, Río Negro). Esta especie es extremadamente plástica por lo cual la forma de sus valvas está influenciada por las condiciones ambientales. Seed (1980) describe que para los moluscos en general, y mitílidos en particular, la forma definitiva de la valva depende de varios factores: restricciones filogenéticas, adaptación, limitaciones estructurales vinculadas a los materiales disponibles y los efectos ecofenotípicos o plasticidad fenotípica promovida por condiciones ambientales. Estos efectos producen, en el caso del mejillón, que aquellos individuos provenientes del intermareal tengan valvas más gruesas y redondeadas y la impronta del 


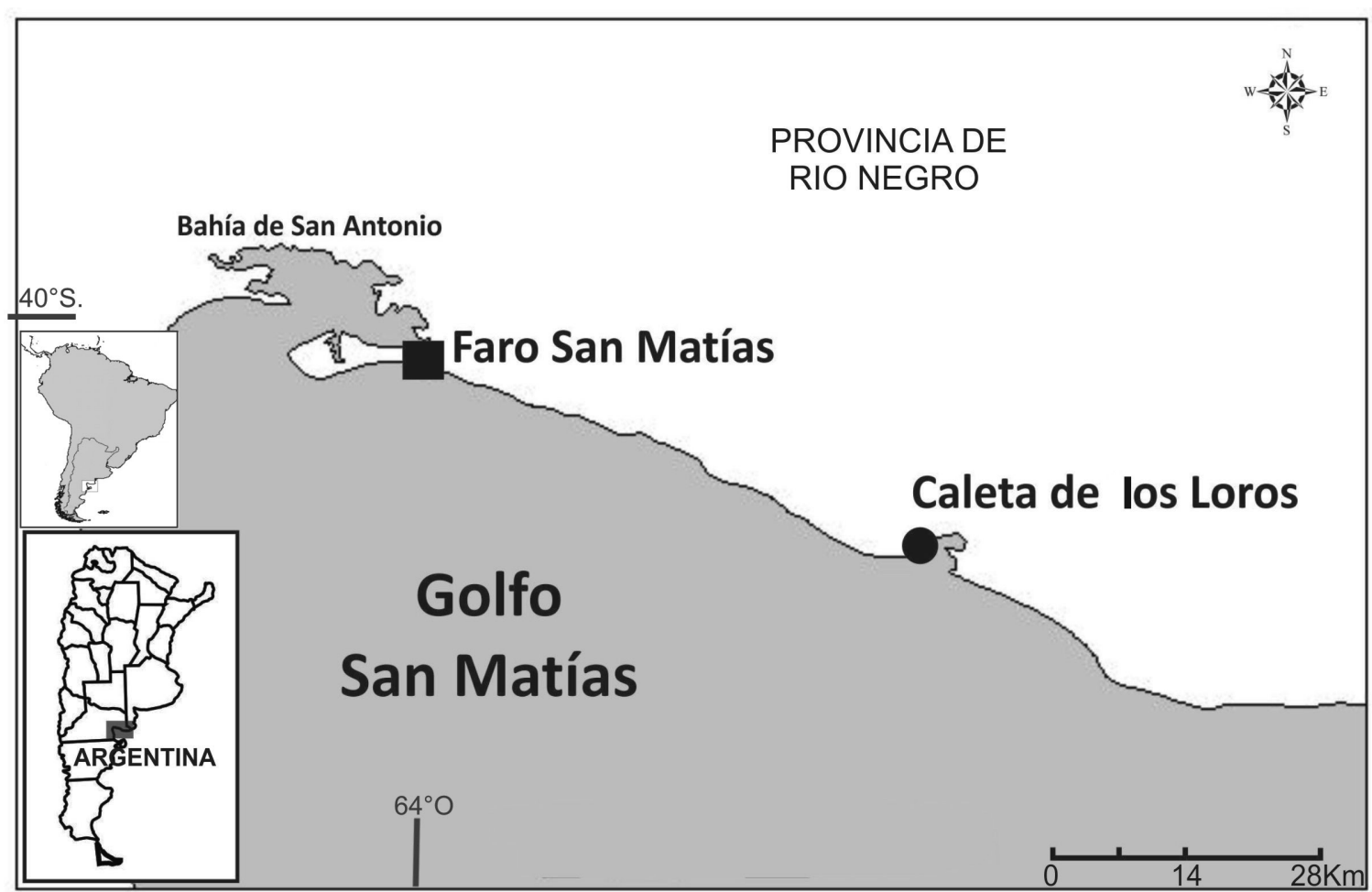

Fig. 1. Ubicación de la localidad arqueológica Faro San Matías y zona de colecta actual de Caleta de los Loros.

músculo aductor posterior más grande que en los provenientes de ambientes submareales (Savoya, 2012). Por otro lado, individuos procedentes de sitios con alto grado de solapamiento espacial tienen valvas más elongadas. En el golfo San Matías, M. platensis forma bancos densos en ambientes submareales, mientras que en el intermareal su abundancia es mucho menor. Como consecuencia, la plasticidad fenotípica de M. platensis puede ser reflejada en las relaciones alométricas que vinculan dos medidas de la valva. Para ello, se analizan estas relaciones a partir de la distribución de frecuencia de tamaños de valvas enteras colectadas en la actualidad de la zona intermareal y submareal de la localidad Caleta de los Loros y los mejillones procedentes de diferentes concheros de FSM (Fig. 1).

\section{ÁREA DE ESTUDIO Y ANTECEDENTES}

El golfo San Matías es el mayor de los denominados golfos norpatagónicos (Fig. 1). La región se destaca por un clima semiárido con temperaturas medias de $15^{\circ} \mathrm{C}$ y lluvias anuales menores a los $300 \mathrm{~mm}$. Se caracteriza por un régimen macromareal, con mareas semidiurnas de alturas medias de 7,62 m y máximos de sicigias de 9,19 m en el puerto de San Antonio Este, en la bahía de San Antonio (Servicio de Hidrografía Naval, 2017). Esta altura de marea es la segunda en el Atlántico sud-occidental y genera una amplia franja intermareal que representa la transición entre el ambiente terrestre y marino. La zona que permanece siempre sumergida se denomina submareal o infralitoral.

El flujo y reflujo producido durante el ciclo de mareas sobre una extensa superficie de baja pendiente, como es el caso en esta parte del golfo, afecta el entorno circundante, generando una marcada estacionalidad térmica en el agua de mar. Esto es especialmente visible en sitios como la bahía de San Antonio (BSA) o la Caleta de los Loros (CL), donde pueden encontrarse organismos actuales de Mytilus platensis tanto en el intermareal como en el submareal.

El contexto geomorfológico de la localidad arqueológica Faro San Matías (FSM), en la zona aledaña a la BSA, corresponde a dunas que se 
apoyan sobre una terraza marina pleistocena de unos $15 \mathrm{~m}$ de altura (Kokot \& Favier Dubois, 2017). En la actualidad esta terraza conforma un paleoacantilado separado de la playa actual por una faja poco extensa $(50 \mathrm{~m})$ de cordones litorales y dunas. Una relativa estabilidad de la línea de costa en este sector desde comienzos del Holoceno tardío habría permitido que ocupaciones diacrónicas se hallen muy cercanas en el espacio sobre este paleoacantilado (Favier Dubois \& Borella, 2007; Borella et al. 2016). Durante estudios previos en esta localidad se excavaron concheros que representan múltiples eventos de descarte de valvas, a los que se asocian restos de mamíferos marinos y terrestres, escasos peces, instrumentos líticos y fragmentos de cerámica (Borella et al. 2011, 2016). Los fechados disponibles para estos concheros permitieron acotar a unos 1500 años el rango de ocupación de este espacio, esto es, entre los ca. 2.910 y 1.380 años ${ }^{14} \mathrm{C}$ AP (Borella et al. 2016) (Tabla 1). En este litoral norte del golfo San Matías, las acumulaciones de exoesqueletos de moluscos en los concheros están compuestas principalmente por Mytilus platensis y Aulacomya atra (cholgas), en menor medida Brachidontes rodriguezi (mejillines) y Crepidula spp. (botecitos). Estos concheros presentan una extensión variable de entre 1 a $10 \mathrm{~m}$ de largo y un espesor que normalmente no supera los $30 \mathrm{~cm}$ (Favier Dubois \& Borella, 2007).

Estudios geoarqueológicos y tafonómicos previos acerca de la estructura y composición de los concheros de la costa norte del GSM, han permitido inferir una elevada resolución e integridad en los mismos y reconocer eventos puntuales de consumo/descarte de mitílidos en muchos casos (Favier Dubois \& Borella, 2007; Favier Dubois et al. 2015). La presencia de carbones de mayor tamaño en la base de los concheros ha indicado que la acumulación comenzaría con el encendido de un fuego sobre el que luego se arrojan los moluscos para su apertura y cocción (ver Favier Dubois \& Borella, 2007). El aporte reiterado de valvas habría generado una estructura de acumulación que se ha visto sepultada de forma rápida por las arenas eólicas del ambiente costero (Favier Dubois \& Borella, 2007), hecho que habría favorecido la preservación de las mismas y la recuperación de una gran cantidad de especímenes enteros para este estudio.

\section{METODOLOGÍA}

El material malacológico actual fue recolectado durante un relevamiento realizado en la población de mejillones situada en el interior de CL. El mismo consistió en la colecta de 715 individuos en el sector intermareal y 598 en el sector submareal somero. De ambos conjuntos se obtuvieron submuestras de 108 individuos cada una, en las cuales se tomaron las siguientes medidas: longitud (mayor distancia desde el umbo al margen, $L t$ ), espesor (mayor distancia entre valvas, Es) y alto máximo (mayor distancia perpendicular a las otras dos, $A t$ ), utilizando un calibre digital con una precisión de $0.1 \mathrm{~mm}$. También se estimó el

Tabla 1. Cronología de los sondeos analizados en la localidad FSM.

Tomado de Favier Dubois et al. (2009) y Borella et al. (2016). Todas las dataciones sobre valvas se realizaron en Mytilus platensis. Entre paréntesis se expresa la media de la edad calibrada.

\begin{tabular}{|c|c|c|c|c|}
\hline Sitio arqueológico & Material datado & $\begin{array}{c}\text { Edad }{ }^{14} \mathrm{C} \\
\text { Obtenida (edades AP) }\end{array}$ & $\begin{array}{c}\text { Rango calibrado } \\
\text { (edades AP) }\end{array}$ & $\begin{array}{c}\text { Referencia de } \\
\text { Laboratorio }\end{array}$ \\
\hline FSM S2 & Carbones & $2.910 \pm 90$ & $\begin{array}{l}2.869-3.141 \\
(3.004)\end{array}$ & LP-1878 \\
\hline FSM S5/6 & Carbones & $1.380 \pm 90$ & $\begin{array}{c}1.112-1.345 \\
(1.236)\end{array}$ & LP-1873 \\
\hline FSM S12 & Valvas & $2.460 \pm 80$ & $\begin{array}{l}2.127-2.376 \\
\quad(2.269)\end{array}$ & LP-3159 \\
\hline FSM M I & Valvas & $1.680 \pm 90$ & $\begin{array}{l}1.271-1.480 \\
\quad(1.372)\end{array}$ & LP-2352 \\
\hline FSM M II & Valvas & $1.630 \pm 70$ & $\begin{array}{c}1.230-1.404 \\
(1.319)\end{array}$ & LP-2358 \\
\hline
\end{tabular}


volumen interno de la cavidad intervalvar $(V i)$ del siguiente modo: a) se registró el volumen total registrando el peso del agua desplazada al ser sumergido en un recipiente; b) una vez removida la carne se registró el volumen de las valvas; c) por diferencia de ambos se estimó el volumen interno.

Las relaciones alométricas entre estas variables se estimaron mediante el ajuste de modelos lineales $(\mathrm{Y}=\mathrm{a}+\mathrm{bX})$ en el caso de variables unidimensionales (At $y$ Es us Lt) $y$ un modelo potencial $(\mathrm{Y}=\mathrm{a}$. $\mathrm{Xb}$ ) en el caso del volumen interno (Vi vs Lt). Los ajustes se realizaron mediante el método de máxima verosimilitud y los contrastes de las mismas entre las dos procedencias se realizaron mediante el método del cociente de verosimilitud. Estas relaciones ofrecen valores de referencia para una procedencia conocida, a partir de los cuales se llevó a cabo el estudio comparativo entre muestras actuales y arqueológicas.

El material arqueológico seleccionado para este análisis proviene de las excavaciones llevadas a cabo en la localidad FSM en los años 2006, 2007 y 2009. Con el objetivo de estudiar las zonas de colecta de mejillones durante el Holoceno tardío, se analizaron específicamente las valvas enteras de M. platensis. Las valvas provienen de diez concheros identificados en sondeos de la localidad FSM: sondeos 2 a 8, 12, montículos I (MI) y II (MII). En total se relevaron 21 niveles de excavación de 10 loci diferentes.

Las relaciones alométricas entre valvas enteras de diferentes concheros se estudiaron de modo idéntico al material malacológico actual recuperado en CL. Las relaciones alométricas entre variables se estimaron mediante un ajuste de regresión lineal y se compararon mediante test estadísticos de paralelismo de pendientes que permitieron estimar un valor para cada grupo de datos (sondeo). Para completar la inferencia sobre la procedencia de las valvas en cada conchero se incorporaron los registros de las variables morfométricas de cada loci a la base de datos malacológicos de procedencia conocida y se procedió a un análisis multivariado discriminante (AD) canónico. Este método permite: 1) discriminar, en base a las variables seleccionadas, grupos definidos a priori y poder representar las observaciones en un espacio donde las diferencias entre grupos sean máximas y 2) clasificar nuevos casos en los grupos establecidos a priori sobre la base de una regla de clasificación basada en las variables independientes.

Por otra parte, se analizó la composición biogénica total de los concheros de FSM, teniendo en cuenta otras especies marinas asociadas a los moluscos y sus características ecológicas, a fin de agregar otra variable a tener en cuenta en los análisis de procedencia. Las edades radiocarbónicas publicadas previamente fueron calibradas para este trabajo usando el programa CALIB 7.1 (Hogg et al. 2013; Reimer et al. 2013) a 1 sigma. Para las muestras continentales (carbones) se utilizó la curva del Hemisferio sur SHcal 13 y para las muestras marinas (valvas) la curva Marine 13. En este último caso se introdujo un $\Delta \mathrm{R}$ de $-134 \pm 51$ de acuerdo al valor de efecto reservorio obtenido localmente para la costa rionegrina (Favier Dubois, 2009) (Tabla 1).

\section{RESULTADOS}

\section{Valores de referencia actuales}

La estructura de tallas de los sectores intermareal y submareal del banco de mejillones actuales de CL resulta diferente. Los individuos recolectados en el intermareal tienen un rango de tallas entre 1 y $62 \mathrm{~mm}$ de largo de valva, mientras que en los colectados en el submareal somero entre 17 y 109 mm (Fig. 2).

Las relaciones alométricas entre las cuatro variables medidas en las valvas de los dos ambientes (Tabla 2), revelan que las provenientes del intermareal tienen mayor espesor y volumen interno o intervalvar para un mismo largo o alto. En cambio, la relación Lt-At no presenta diferencias significativas entre ambientes. Sin embargo, el patrón de variación morfométrico permite estimar la zona de procedencia de cualquier conjunto de valvas de origen desconocido.

\section{Valvas arqueológicas de la localidad FSM}

Se analizan las relaciones alométricas de un total de 932 valvas arqueológicas enteras de la localidad FSM. La mayor potencia estratigráfica se registró en el loci S5, en el cual se excavaron 4 niveles (Tabla 3). 

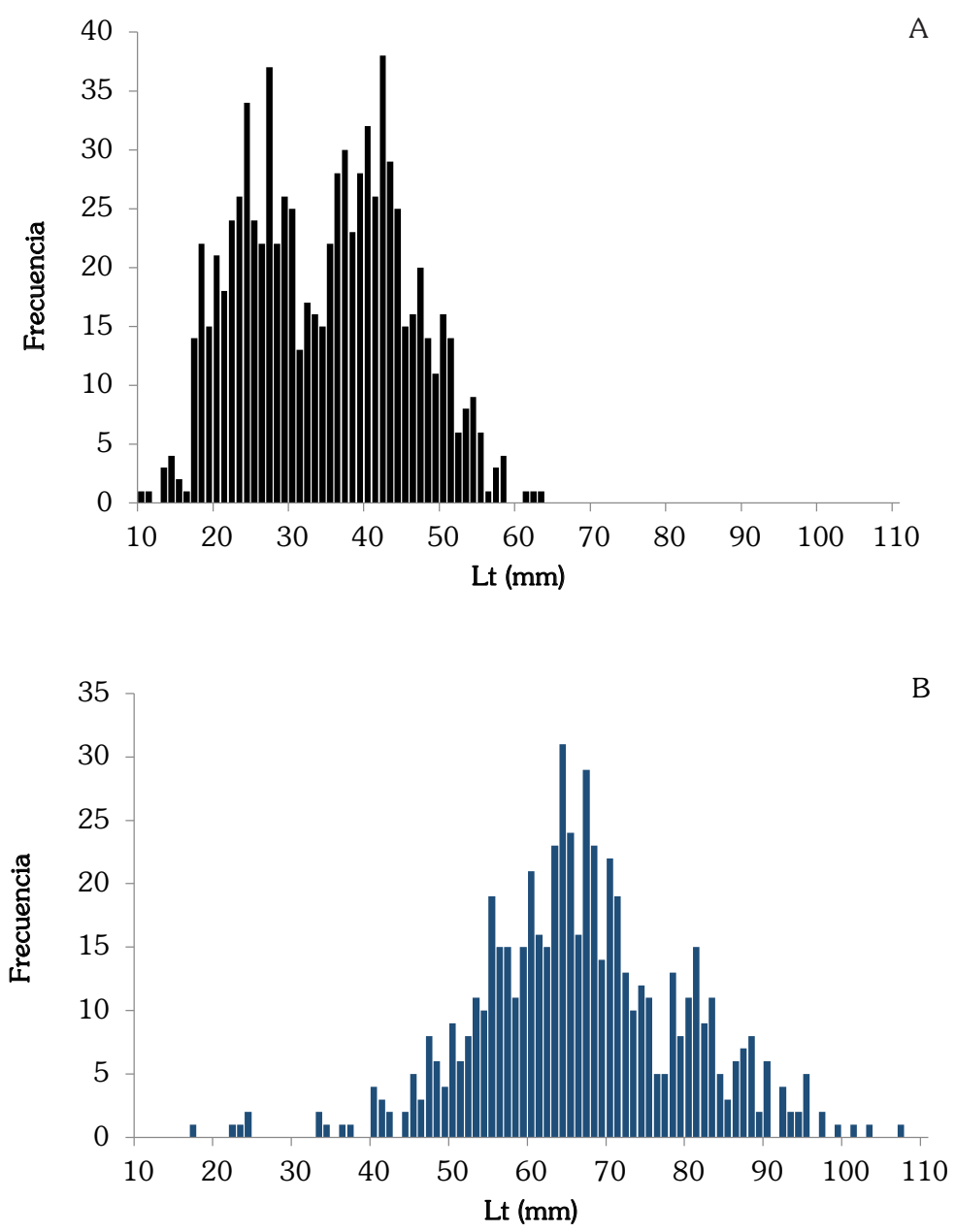

Fig. 2. Distribución de frecuencias de tallas de mejillones del intermareal (A) y submareal (B) de CL.

Tabla 2. Relaciones alométricas de mejillones recolectados en el intermareal y submareal somero de CL. La cuarta columna denota el nivel de significancia estimado por el test de cociente de verosimilitud.

\begin{tabular}{|c|c|c|c|}
\hline & Intermareal & Submareal & $\mathrm{P}$ \\
\hline Alto - Largo & $A t=3.1164+0.4654 . L t$ & $A t=4.2144+0.4377 . L t$ & NS \\
\hline Espesor - Largo & $E s=-0.7594+0.5146 . L t$ & $E s=-0.274+0.405 . L t$ & $<0.01$ \\
\hline Volumen interno - Largo & $V i=0.000195 . L t^{2.789}$ & $V i=0.000146 . L t^{2.816}$ & $<0.01$ \\
\hline Espesor - Alto & $E s=-2.85+1.047 . A t$ & $E s=-3.045+0.9 . A t$ & $<0.01$ \\
\hline
\end{tabular}

Las valvas recolectadas en FSM presentan un rango de valores de Lt entre 20 y $70 \mathrm{~mm}$. Los valores mínimos varían desde $27.83 \mathrm{~mm}$ (S6) hasta $41.35 \mathrm{~mm}$ (S3) y los Lt máximos varían entre 53.74 $\mathrm{mm}$ (S12) y $77.79 \mathrm{~mm}$ (S5). La mayor diversidad de tallas se registra en S5 con una variabilidad entre Lt mínimo y máximo de $49.5 \mathrm{~mm}$ (Tabla 3). El análisis combinado de las variables morfométricas At/Es, referidas a la proporción de alto y espesor, indica que las relaciones se mantienen relativamente 
Tabla 3. Cantidad de valvas enteras recuperadas por sondeos en FSM y relaciones alométricas promediadas por nivel de excavación: longitud (Lt), alto máximo (At) y espesor (Es).

\begin{tabular}{ccccccc}
\hline $\begin{array}{c}\text { Loci } \\
\text { FSM }\end{array}$ & ${ }^{14} \mathrm{C} \mathrm{AP}$ & $\mathrm{N}$ & $\begin{array}{c}\text { Total de } \\
\text { niveles }\end{array}$ & Mín & Máx & Media \\
\hline S2 & $2.910 \pm 90$ & 38 & 1 & 34.14 & 70.83 & 52.49 \\
S3 & - & 50 & 1 & 41.35 & 65.33 & 53.34 \\
S4 & - & 167 & 2 & 30.21 & 60.80 & 45.51 \\
S12 & $2.460 \pm 80$ & 146 & 2 & 29.16 & 53.74 & 41.45 \\
MII & $1.630 \pm 70$ & 63 & 1 & 36.32 & 68.22 & 52.27 \\
MI & $1.680 \pm 90$ & 45 & 1 & 33.63 & 70.22 & 51.93 \\
S5 & - & 344 & 4 & 28.25 & 77.79 & 53.02 \\
S6 & $1.380 \pm 90$ & 25 & 3 & 27.83 & 60.78 & 44.31 \\
S7 & - & 27 & 3 & 35.63 & 75.50 & 55.57 \\
S8 & - & 27 & 3 & 27.93 & 71.88 & 49.91 \\
\hline \multicolumn{7}{c}{ Total } \\
\hline
\end{tabular}

constantes en la mayoría de los sondeos, excepto en S5 donde se registran los mayores tamaños y una morfología más globosa.

En todos los sondeos, las valvas alcanzan un Lt máximo mayor o igual a $60 \mathrm{~mm}$ de talla, cuyo valor es el promedio para uso comercial en la actualidad. Una excepción se observa en S12 con 53 mm de Lt máximo. Sin embargo, una tendencia común que se registra en los diferentes loci de FSM es que las frecuencias de talla entre 37 a $46 \mathrm{~mm}$ son las más abundantes y una menor proporción corresponde a valores entre 47 y $56 \mathrm{~mm}$ (Fig. 3).

Desde una perspectiva temporal, se comparan los datos de frecuencias de tallas provenientes de concheros con cronologías más tempranas y más tardías. A partir de esto, es posible observar valvas de mayor tamaño de talla $(\geq$ $57 \mathrm{~mm}$ ) en los sondeos con cronologías entre ca. 1600 y 1300 años AP en comparación con los de edades previas, entre ca. 2.900 y 2.200 años AP (Fig. 4). La tendencia observada entre ambos grupos temporales podrá ser discutida en futuros estudios que los vinculen con tamaños disponibles para su recolección o bien con problemas relacionados con la preservación/conservación de los exoesqueletos del conjunto analizado.

\section{Zonas de colecta en la localidad FSM}

Los resultados de los test estadísticos de comparación de rectas de regresión de EspesorLargo de valva de los sondeos S2, S6 y S12 indican semejanzas con los datos alométricos de valvas procedentes de la zona submareal y disparidad con todos los del ambiente intermareal. El análisis discriminante canónico asigna un mayor porcentaje de mejillones submareales en todos los casos (Tabla 4).

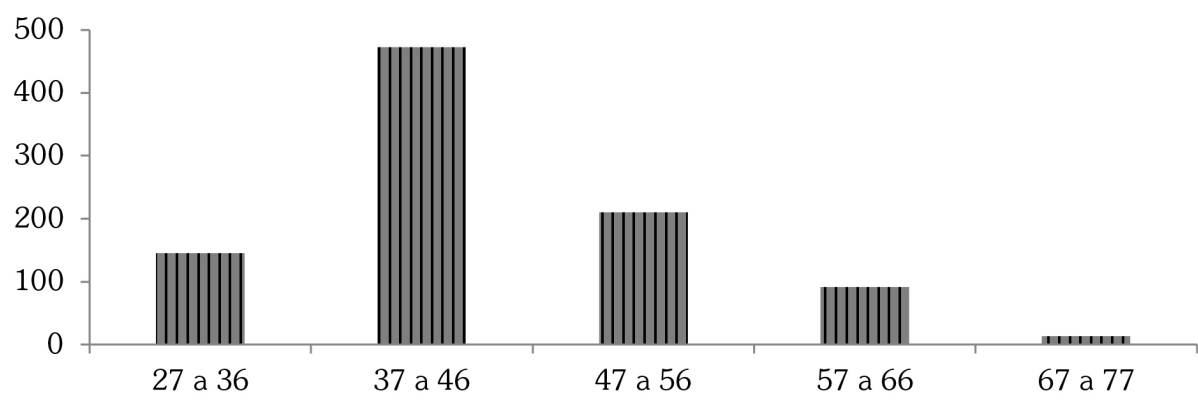

Fig. 3. Frecuencia de tallas de valvas enteras recuperadas en la localidad FSM. 


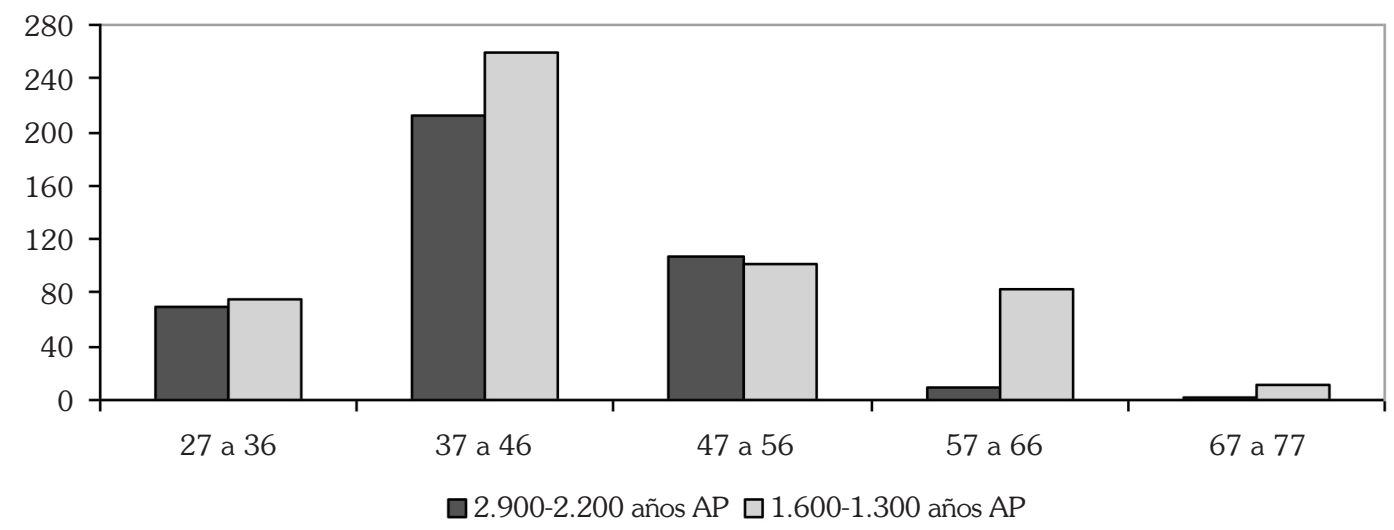

Fig. 4. Frecuencia de Lt máximas de M. platensis en FSM de acuerdo a dos bloques temporales del Holoceno tardío (temprano y tardío).

Tabla 4. Resultados de la inferencia sobre la procedencia de las valvas arqueológicas en los sondeos de FSM, a partir de la comparación de rectas de regresión (Espesor - Largo) y el análisis discriminante canónico. El valor de $\mathrm{P}$ representa la probabilidad de igualdad de rectas de cada sondeo con la de cada ambiente conocido. El porcentaje representa a los individuos asignados por el análisis discriminante canónico a cada origen de acuerdo a todas las variables morfométricas.

\begin{tabular}{cccccc}
\hline & \multicolumn{3}{c}{$\begin{array}{c}\text { Comparación de rectas de regresión } \\
\text { (Es - Lt) }\end{array}$} & \multicolumn{2}{c}{ Análisis discriminante canónico } \\
& & Submareal & Intermareal & Submareal & Intermareal \\
\hline Sondeo & $\mathrm{N}$ & $\mathrm{P}$ & $\mathrm{P}$ & $\%$ & $\%$ \\
S2 & 38 & $<0.01$ & 0.911 & 34.2 & 65.8 \\
S3 & 50 & $<0.01$ & $<0.01$ & 4.0 & 96.0 \\
S4 & 167 & $<0.01$ & $<0.01$ & 14.4 & 85.6 \\
S5 & 344 & $<0.01$ & $<0.01$ & 27.3 & 72.7 \\
S6 & 25 & $<0.01$ & 0.59 & 34.6 & 65.4 \\
S7 & 27 & $<0.01$ & $<0.01$ & 3.8 & 96.2 \\
S8 & 27 & $<0.01$ & $<0.01$ & 7.4 & 92.6 \\
S12 & 146 & $<0.01$ & 0.05 & 34.9 & 65.1 \\
MI y MII & 108 & $<0.01$ & $<0.01$ & 27.4 & 72.6 \\
\hline
\end{tabular}

En los concheros de FSM se analizan complementariamente los restos de otras valvas de mitílidos (cholgas y mejillines). Las cholgas se presentan en la zona intermareal y hasta una profundidad de $30-50 \mathrm{~m}$, en sustratos duros al igual que los demás mitílidos y en algunos ambientes protegidos en sustratos arenosos y fangosos. En la actualidad, los bancos de cholgas, explotados por buzos marisqueros, normalmente se encuentran entre los 5 y los $20 \mathrm{~m}$ de profundidad (Zaixso et al. 2015).

\section{Especies asociadas}

El balano identificado en los concheros es Austromegabalanus psittacus (Cirripedia, Megabalaninae) y presenta una variabilidad de tamaños entre $8 \mathrm{~mm}$ y $30 \mathrm{~mm}$ de longitud (Fig. 5). Se trata de una especie que comúnmente se denomina "Picoroco". Se encuentra en pozas de marea del intermareal inferior y zona submareal. Es decir que para su crecimiento requiere estar sumergido hasta profundidades comprendidas entre 2 y $4 \mathrm{~m}$, las 
cuales serían suficientes para alcanzar los mayores tamaños (Lanas et al. 2011; López et al. 2012; Pappalardo et al. 2016). Este crustáceo se adhiere firmemente al sustrato (rocas, maderas, moluscos, otros crustáceos, etc.). Las cholgas recuperadas en los sondeos 2, 5, 6, 7, 8 y 12 presentan adheridas a su superficie este crustáceo. En FSM, sondeos 2, 5 y 8 , se recuperan ejemplares sueltos de A. psittacus, con la impronta de cholgas en su superficie de adhesión (llamada base).

Por otro lado, se identificaron tubos de poliquetos de la familia Serpullidae (Annelida, Polychaeta) adheridos a Aulacomya atra. Los mismos son muy comunes como epibiontes en bancos de mejillones de la zona intermareal $y$ submareal somera (Diez et al. 2016). En FSM se registran tubos calcáreos cilíndricos de poliquetos en S6 y S12 sobre A. atra. Estudios recientes llevados a cabo en el área (Diez et al. 2011, 2016) sugieren una correlación positiva entre valvas de mayor longitud de A. atra, y mayor batimetría, como factores que favorecen la infestación de serpúlidos. Asimismo, descartan que la presencia de poliquetos se restrinja a zonas poco profundas, ya que los mismos se registran a $15 \mathrm{~m}$ de profundidad (Diez et al. 2016).

\section{Zona de colecta y estacionalidad}

Como parte de este estudio se analiza la relación entre la zona de colecta y la estacionalidad de captura. Para esto se utiliza información de un trabajo previo realizado en la costa norte del GSM que incluyó los sondeos analizados para determinar procedencia (Steffan \& Morsan, 2015). En ese trabajo se observa que los distintos niveles de un mismo conchero indicaban la misma estacionalidad de colecta. Ésta se infiere a partir de la observación de los bordes externos de los ejemplares, si son opacos la colecta corresponde a meses cálidos, y si son translúcidos, a meses fríos. La mayoría de los bordes externos opacos de $M$. platensis se registra en los sondeos: 5, 6, 7, 8 y MI (Fig. 6). En consecuencia, la colecta de los mismos correspondería a los meses cálidos (octubre a marzo). Mientras que en los sondeos 2, 3, 4, 12 y MII, se infiere que la colecta se habría llevado a cabo durante los meses fríos de fines de otoño a primavera (mayo a septiembre).

Cronológicamente se observa que entre 3.004 y 1.372 años cal. AP se explotaron los moluscos de la especie Mytilus platensis en invierno, mientras entre 1.372 y 1.236 años cal.

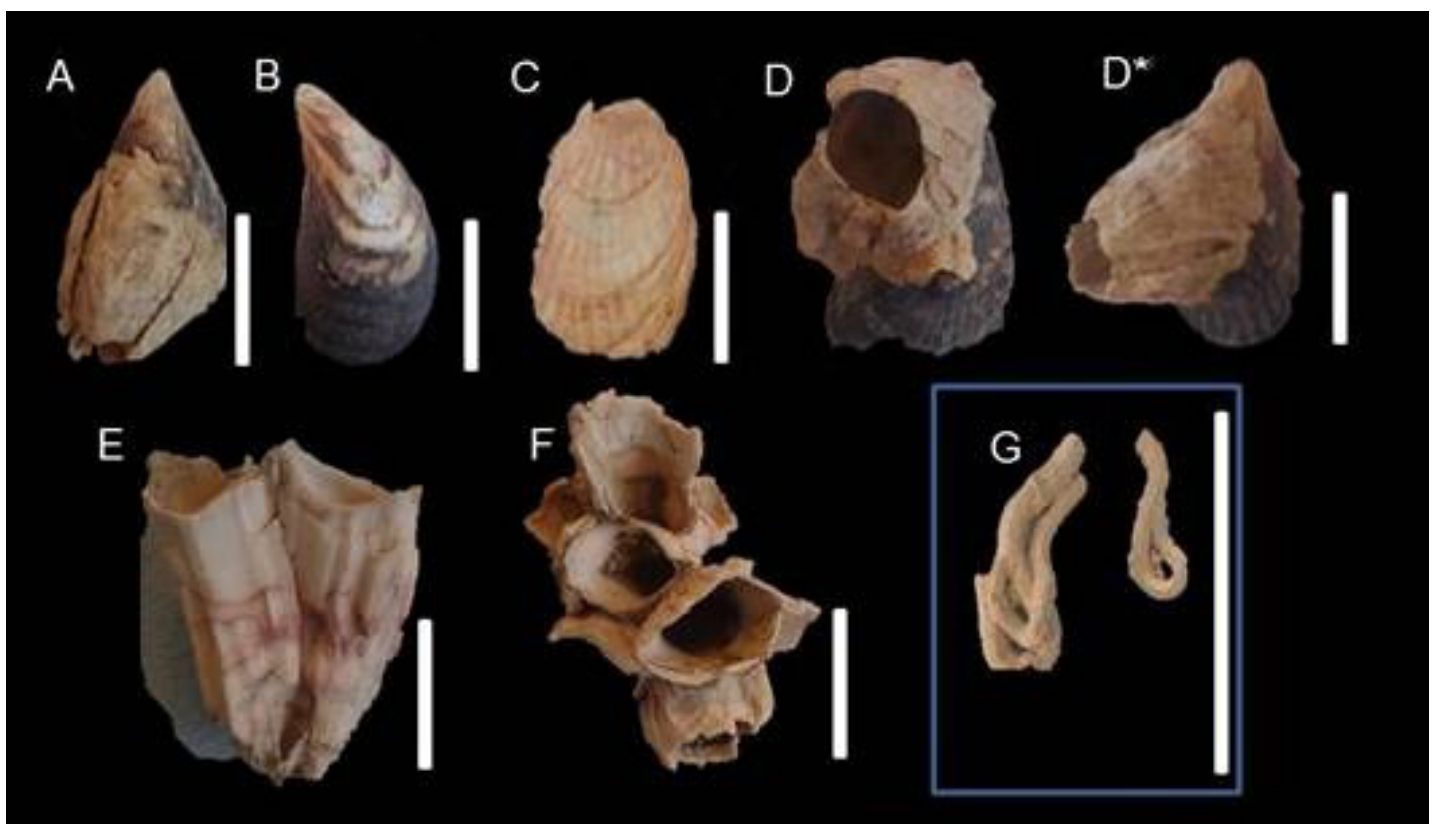

Fig. 5. Taxones asociados a M. platensis en la localidad FSM. A. Aulacomya atra y Austromegabalanus psittacus adherido; B. (negativo de superficie de adhesión); C. (negativo de A. atra en la base de A. psittacus); D. (vista apical) y D* (vista lateral); E. (vista lateral) y F. (vista apical); G. Serpúlidos. La escala indica $5 \mathrm{~cm}$. 


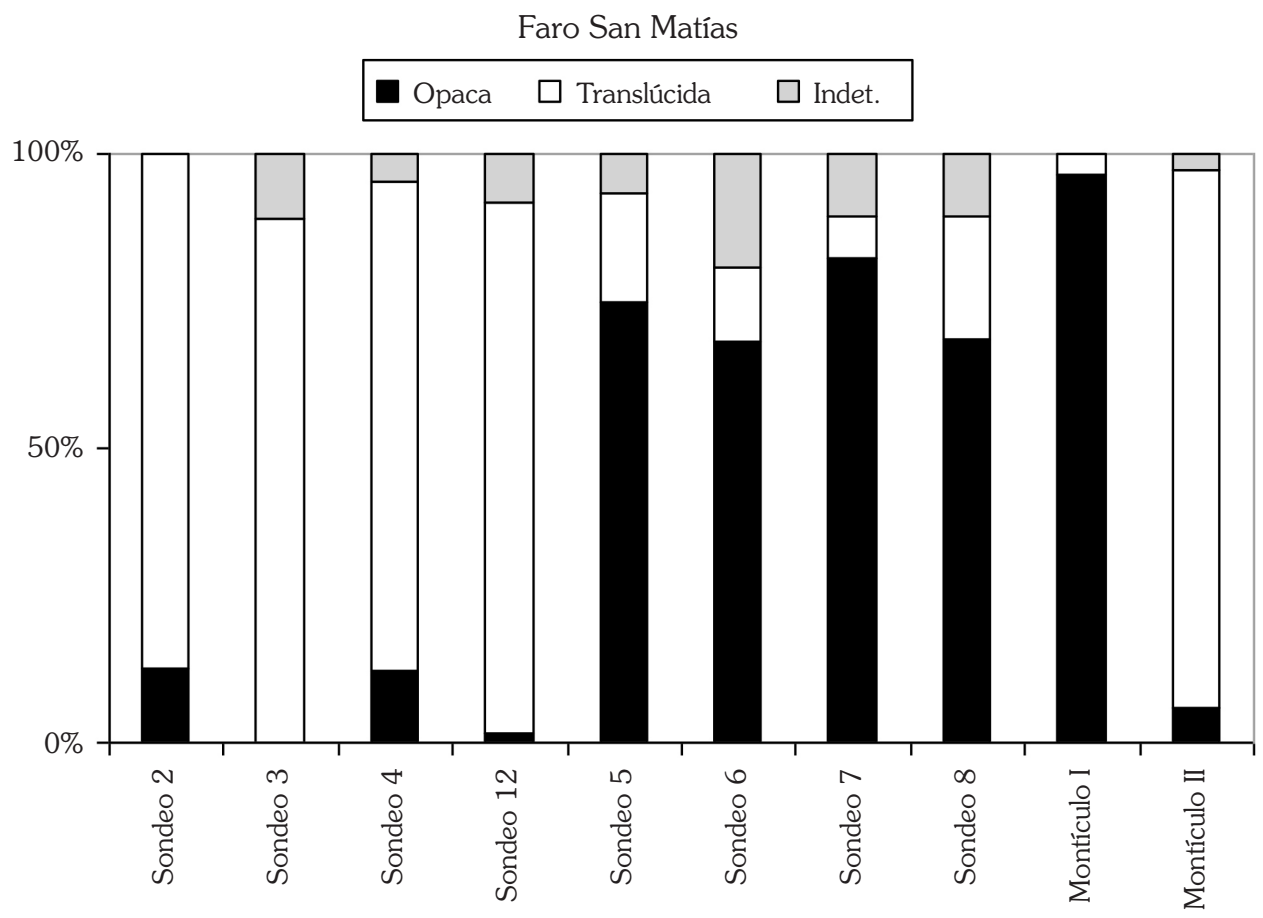

Fig. 6. Abundancias relativas de bordes externos translúcidos y opacos en Mytilus platensis en sondeos de FSM.

AP las colectas se llevaron a cabo durante los meses más cálidos. Además, esto coincide con una mayor abundancia de las valvas enteras recuperadas en FSM correspondientes a recolecciones realizadas durante el verano. Esta situación podría sugerir una mayor cantidad de moluscos necesarios para el consumo o bien un sesgo metodológico en la recolección/preservación de la muestra. En consecuencia, no se observa una relación directa entre la preferencia de una determinada zona de recolección y la estacionalidad. Las inmersiones a mayor profundidad para la recolección de los moluscos se habrían llevado a cabo independiente de la estacionalidad, ya que ocurrieron tanto durante los meses cálidos (S6, S7 y S8) como fríos (S2 y S12).

\section{DISCUSIÓN}

A partir de los resultados obtenidos en los concheros de la localidad arqueológica FSM se discuten las zonas de colecta y los modos de aprovisionamiento de los mitílidos en la costa a partir de tres ejes de análisis. Luego se integran a la discusión general de la explotación de los recursos costeros en FSM.

1) ¿La recolección de Mytilus platensis se llevó a cabo solamente en la zona intermareal o ha incluido también el sector submareal somero?

En primer lugar, es importante destacar que la distinción entre mejillones colectados en un ambiente intermareal o submareal está fuertemente condicionada por la versatilidad morfológica que esta especie presenta, promovida por factores ambientales. Entre éstos, el hecho de permanecer descubiertos durante periodos prolongados de tiempo, con alimentación reducida y expuestos a cambios extremos de temperatura, es el efecto más relevante en la determinación de la forma definitiva de la morfología de las valvas. Las especies del género Mytilus son especialmente plásticas en respuesta a estas condiciones. En $M$. platensis la fuente de mayor variabilidad está dada por la forma de crecimiento en el intermareal que tiende a ser más globosa por incremento del espesor o distancia entre las valvas. 
En segundo lugar y en relación a la pregunta inicial, el material arqueológico recogido en los concheros presenta una amplia variabilidad morfológica individual, la cual se manifiesta en la dificultad de encontrar una prueba estadística categórica para asignar un origen. Sin embargo, los test multivariados utilizados en este trabajo han aportado la mejor aproximación para abordar el origen de los restos de mejillones arqueológicos, determinando una predominancia en la procedencia submareal.

En algunos sondeos (S2, S6 y S12) los mejillones podrían tener mezcla de procedencia de ambos ambientes. Otro factor que dificulta la interpretación está dado en que en algunos sondeos se registra amplia variabilidad de tamaños de las valvas arqueológicas y por tal motivo no es factible discriminar una clara tendencia en relación a la zona de colecta, pudiendo ser ambas. Esto también podría relacionarse con la práctica de recolección de esta especie en racimos, es decir, sin una selección individual de los ejemplares por tamaños como han mencionado Orquera y Piana (2001) para la costa norte del canal Beagle (Argentina). De este modo, la variabilidad de tamaños se vincularía en ocasiones con una modalidad de colecta poco selectiva y no directamente con zona de recolección de los invertebrados. Con la finalidad de aportar datos a la discusión, se analizó y exploró la ecología de la fauna acompañante referida a los restos de balano, tubos de poliquetos y cholgas, teniendo en cuenta que son materiales asociados a $M$. platensis en algunos sitios (ej.: en S5).

Los balanos presentan una estructura adhesiva que puede variar filogenéticamente; sin embargo, su adherencia depende de la interacción de las propiedades físicas y químicas del adhesivo y del sustrato. Asimismo, los crustáceos adheridos a sustratos duros naturales en la zona intermareal o submareal también experimentan arrastre que pueden conducir a desprendimientos de la base (Denny et al. 1985). Teniendo en cuenta el tamaño de los balanos recuperados en FSM y los negativos de adhesión sobre $A$. atra, se infiere una procedencia submareal, más allá de las zonas sujetas a las variaciones entre pleamar y bajamar (intermareal).

En el caso de los poliquetos también presentan fuertes adherencias a diversas superficies duras. Los restos recuperados en concheros parecen haber ingresado al registro adheridos a cholgas. Las características ecológicas de los bivalvos y otros bioindicadores requieren de sustrato duro o rocoso, por tal motivo es factible inferir que las zonas de colecta se vinculan en general con este tipo de sustrato. Otra alternativa correspondería a los bancos mixtos de cholga-mejillón, desarrollados en fondos blandos y bajo condiciones de estabilidad; en estos casos los mejillones evolucionan junto a las cholgas (Zaixso, 2004).

La explotación de la zona intermareal ha sido destacada para diferentes sectores costeros de la Patagonia y Tierra del Fuego argentinas (Piana \& Estévez Escalera, 1995; Zubimendi et al. 2005; Savoya et al. 2015), o bien el intermareal medio e inferior y submareal somero en Tierra del Fuego (Orquera \& Piana, 2001). Otra hipótesis alternativa para la colecta de Mytilus y Aulacomya ha sido su recolección en condiciones de marea "extraordinaria", como las mareas de sicigia equinocciales u otras mareas máximas, en que las bajamares son más bajas que las alturas medias (Santiago et al. 2014). Sin embargo, el presente análisis permite aportar herramientas de análisis complementarias para obtener información adicional y discutir las zonas de recolección de recursos marinos en aguas más profundas.

2) ¿La zona seleccionada para la colecta tiene alguna relación con la estacionalidad?

Es importante destacar que las valvas provenientes de distintos niveles de un mismo conchero indican la misma estacionalidad. Este fue uno de los criterios utilizados para afirmar que la acumulación de valvas, carbones y restos óseos generados por las actividades humanas, representa eventos sincrónicos de descarte en muchos concheros (Favier Dubois et al. 2015).

Los mayores tamaños de Lt corresponden a valvas colectadas en meses cálidos, en orden decreciente de tamaño corresponden a S5, S7, S8 y MI; excepto en S2 que indica colectas en meses fríos. La amplia variabilidad de tamaños de valvas en S5 parece corresponder a la extracción de diferentes conjuntos de moluscos (o racimos) en una extracción generalizada o poco seleccionada. Una situación diferente se observa en S12 donde 
los tamaños son todos pequeños, esta selección podría corresponder a una baja disponibilidad de tamaños grandes o a mezcla de ambientes (teniendo en cuenta que los mejillones intermareales tienen menor tasa de crecimiento). En síntesis, la zona de colecta inferida en cada uno de los sondeos de FSM no coincide con una estacionalidad en particular, es decir, no cambia la zona de recolección con la estación del año.

Las inmersiones durante los meses fríos habrían obtenido el beneficio de una explotación de este recurso vinculada a su mayor rendimiento proteico de acuerdo con los ciclos de inicio de desove de los mitílidos (Penchaszadeh, 1980); mientras que durante los meses cálidos, su recolección submareal podría estar vinculada con la búsqueda de ejemplares de mayor tamaño. No obstante, y tal como se ha planteado en trabajos previos, es factible sostener que la explotación de los moluscos fue la principal actividad extractiva en esta localidad arqueológica junto con el aprovechamiento de otros recursos, como los mamíferos marinos y peces (Borella et al. 2016).

3) ¿Es posible detectar cambios a través del tiempo en las conductas de recolección de mejillones?

Para explorar las conductas de consumo de recursos costeros es necesario mencionar que estudios faunísticos e isotópicos previos indican dietas predominantemente marinas, con un uso importante de los recursos costeros entre ca. 3.100 y 2.200 años ${ }^{14} \mathrm{C} \mathrm{AP}$; mientras que entre 2.000 y 500 años ${ }^{14} \mathrm{C}$ AP predominan dietas mixtas y continentales, con mayor consumo de recursos terrestres (Favier Dubois et al. 2009; Favier Dubois \& Scartascini, 2012). A partir del presente análisis es importante discutir en términos arqueomalacológicos el aporte de los moluscos en la alimentación de los grupos costeros. En general se le otorga un valor secundario al consumo de moluscos en las dietas marinas, más bien vinculado a su aporte proteico, especialmente en momentos previos al desove (Zubimendi et al. 2005 y citas allí mencionadas). Sin embargo, en la localidad FSM la explotación de los recursos marinos estuvo principalmente orientada a los moluscos y los mamíferos marinos (otáridos y, en menor medida, los cetáceos) (Scartascini, 2014; Borella et al. 2016).

La muestra analizada ha sido dividida en dos grupos cronológicos (tempranos vs tardíos) y de estacionalidad. El grupo 1 (temprano) con edades entre 30.04 y 2.269 años cal. AP, corresponde a los sondeos 2, 3, 4 y 12 (Tabla 1). La colecta se llevó a cabo entre mayo y septiembre. El grupo 2 (tardío) con edades entre 1.372 y 1.236 años cal. $\mathrm{AP}$, corresponde a los sondeos 5, 6, 7, 8 y MI, donde la recolección de los mejillones ocurrió entre octubre y marzo, excepto en el sondeo MII (1319 años cal. AP) en que la recolección indica meses fríos (entre mayo y septiembre).

En relación a la pregunta planteada, si es posible detectar un cambio a través del tiempo, las recolecciones de los mejillones más tempranas corresponden a meses fríos. En las tardías se registran también meses fríos. Sin embargo, de acuerdo a los datos cronológicos disponibles, a partir de 1.372 años cal. AP en FSM la explotación de moluscos se llevó a cabo principalmente durante los meses más cálidos. En cuanto a las zonas de colecta no se observan cambios a través del tiempo, ya que la zona submareal se utilizó desde el inicio de las ocupaciones humanas en FSM hasta los momentos más tardíos, de acuerdo con las edades obtenidas en los sondeos.

\section{CONCLUSIONES}

- Se propone una metodología multivariada que puede utilizarse para discriminar la zona de procedencia intermareal/submareal de especímenes de Mytilus platensis obtenidos en concheros arqueológicos a lo largo del litoral marino patagónico.

- Esta metodología se basa en dos criterios biológicos: 1) un patrón de crecimiento alométrico diferenciable para cada zona, que puede ser discriminado estadísticamente; y 2) el uso de bioindicadores (especies asociadas) que permiten inferir zonas de recolección de manera independiente a partir de requisitos ecológicos.

- Los resultados para FSM indican que entre los 3.004 a los 1.236 años cal. AP en esta localidad se evidencian actividades de recolección de Mytilus platensis tanto en la faja intermareal como submareal, resultando esta última más 
importante, colecta que acompañó la explotación paralela de Aulacomya atra.

- Los datos obtenidos permiten ampliar nuestro conocimiento acerca de las estrategias de explotación de moluscos mitílidos, que hasta el momento se consideraban básicamente colectados en la faja intermareal, en relación con la de otros recursos marinos aprovechados a lo largo del año por parte de los grupos cazadorespescadores-marisqueadores de esta región durante el Holoceno tardío.

\section{AGRADECIMIENTOS}

A la Dra. F. Borella por sus aportes al presente trabajo. A la Dra. Paola Lanas por el asesoramiento de los balanos y a la Dra. Emilia Díaz por su colaboración en la determinación de los poliquetos. Al Centro de Investigación Aplicada y Transferencia Tecnológica en Recursos Marinos "Almirante Storni" (San Antonio Oeste, Río Negro). Este trabajo fue subsidiado por el proyecto PICT-2013 No1128 de la ANPCyT. Agradecemos asimismo a la Unidad Ejecutora INCUAPACONICET (Investigaciones Arqueológicas y Paleontológicas del Cuaternario Pampeano) y a la Facultad de Ciencias Sociales (Olavarría), Universidad Nacional del Centro de la Provincia de Buenos Aires.

\section{BIBLIOGRAFÍA}

Aguirre, M. L., Richiano, S., Álvarez, M. F., \& Eastoe, C. (2009). Malacofauna Cuaternaria del litoral norte de Santa Cruz (Patagonia, Argentina). Geobios, 42, 411-434. https://doi.org/10.1016/j.geobios.2009.01.002

Bar-Yosef Mayer, D. (Ed.). (2005). Archaeomalacology: mollusks in former environments of human behavior. Proceeding of the $9^{\text {th }}$ ICAZ Conference in Durham, 2002. Oxford: Oxbow Books.

Borella, F., Scartascini, F. L., \& Marani, H. (2011). Explorando la subsistencia humana a partir de la evidencia faunística en la costa rionegrina. En F. Borella y Cardillo, M. (coords.), Arqueología de pescadores y marisqueadores en Norpatagonia, descifrando un registro de más de 6000 años (pp. 87-110). Buenos Aires, Argentina: Editorial Dunken.

Borella, F., Cardillo, M., Scartascini, F., Steffan, P., Carranza, E., \& Favier Dubois, C. (2016). Faro San Matías
50 años después: ocupaciones humanas durante el Holoceno tardío (nordpatagonia, Argentina). Revista de Antropología del Museo de Entre Ríos, 2(1), 6276. http://hdl.handle.net/11336/45106

Cakirlar, C. (Ed.). (2011). Archaeomalacology revisited: nondietary use of molluscs in archaeological settings. Proceedings of the archaeomalacology sessions at the 10th ICAZ Conference, Mexico City, 2006. Oxford: Oxbow Books.

Claassen, C. P. (1998). Shells. Cambridge Manuals in Archaeology. Cambridge: University Press.

Denny, M. W., Thomas, L. D., \& Koehl, M. A. (1985). Mechanical limits to size in wave-swept organisms. Ecological Monographs, 55(1), 69-102. https://doi. org/10.2307/1942526

Diez, M. E., Radashevsky, V. I., Orensanz, J. M., \& Cremonte, F. (2011). Spionid polychaetes (Annelida: Spionidae) boring into shells of molluscs of commercial interest in northern Patagonia, Argentina. Italian Journal of Zoology, 78, 497-504. https://doi.org/10.1080/112 50003.2011.572565

Diez, M. E., Vázquez, N., \& Cremonte, F. (2016). Pearls in the ribbed mussel Aulacomya atra caused by polydorin polychaetes (Spionidae) infestation. Journal of Invertebrate Pathology, 140, 42-45. https://doi. org/10.1016/j.jip.2016.08.011

Favier Dubois, C. M. (2009). Valores de efecto reservorio marino para los últimos 5.000 años obtenidos en concheros de la costa atlántica norpatagónica (golfo San Matías, Argentina). Magallania 37(2), 139-147. https://doi. org/10.4067/S0718-22442009000200008

Favier Dubois, C. M., \& Borella, F. (2007). Consideraciones acerca de los procesos de formación de concheros de la costa norte del Golfo San Matías, Río Negro. Cazadores Recolectores del Cono Sur, 2, 151-165.

Favier Dubois, C. M., \& Scartascini, F. (2012). Intensive fishery scenarios on the North Patagonian coast (Río Negro, Argentina) during the Mid-Holocene. Quaternary International, 256, 62-70. https://doi:10.1016/j. quaint.2011.07.041.

Favier Dubois, C. M., Borella, F., \& Tykot, R. H. (2009). Explorando tendencias en el uso humano del espacio $y$ los recursos en el litoral rionegrino (Argentina) durante el Holoceno medio y tardío. En F. Santiago, M. Salemme, M. Álvarez, E. Piana, M. Vásquez \& M. E. Mansur (Eds.), Arqueología de la Patagonia: una mirada desde el último confín (pp. 985-997). Ushuaia: Editorial Utopías.

Favier Dubois, C. M., Borella, F., Steffan, P., \& Ortega, F. 
(2015). Aporte al estudio de la contemporaneidad en las acumulaciones de valvas de origen antrópico en ambientes litorales. Cuadernos del Instituto Nacional de Antropología y Pensamiento Latinoamericano Series Especiales, 2(4), 1-12.

Hammond, H. (2014). Taphonomic analysis of archaeomalacological assemblages: shell middens on the northern coast of Santa Cruz (Patagonia, Argentina). Intersecciones en Antropología Volumen especial, 1, 21-34.

Hammond, H. (2015). Sitios concheros en la costa norte de Santa Cruz: su estructura arqueológica y variabilidad espacial en cazadores recolectores patagónicos. Tesis de Doctorado. Facultad de Ciencias Naturales y Museo. Universidad Nacional de La Plata, Argentina.

Hammond, H., \& Zubimendi, M. A. (Eds.). (2015). Arqueomalacología: abordajes metodológicos $y$ casos de estudio en el Cono Sur, Ciudad Autónoma de Buenos Aires: Fundación de Historia Natural Félix de Azara.

Hammond, H., Zilio, L., \& Zubimendi, M. (2015). Estudio arqueomalacológico en el sitio UNPA. Un caso de reocupación del espacio en la Ría Deseado, Patagonia Argentina. En H. Hammond \& M. A. Zubimendi (Eds.), Arqueomalacología: abordajes metodológicos $y$ casos de estudio en el Cono Sur (pp. 25-58). Ciudad Autónoma de Buenos Aires: Fundación de Historia Natural Félix de Azara.

Hogg, A. G., Hua, Q., Blackwell, P. G., Niu, M., Buck, C. E., Guilderson, T. P., Heaton, T. J., Palmer, J. G., Reimer, P. J., Reimer, R. W., Turney, C. S. M., \& Zimmerman, S. R. H. (2013). SHCal13 Southern Hemisphere Calibration, 0-50,000 Years cal BP. Radiocarbon, 55(4), 1889-1903. https://doi.org/10.2458/azu_js_ rc. 55.16783 .

Kokot, R., \& Favier Dubois, C.M. (2017). Evolución geomorfológica de la bahía de San Antonio, Provincia de Río Negro. Revista de la Asociación Geológica Argentina 74(3), 315-325.

Lanas, P., Riera, M., Kowal, R., López, B. A., \& López, D.A. (2011). Alimentación natural de Austromegabalanus Psittacus (Molina, 1782) (CIRRIPEDIA: BALANIDAE) en el golfo San Jorge (Chubut, Argentina). BioScriba, 4(1), 38-43.

Lobbia, P. (2012). Esclerocronología en valvas de Mytilus spp: análisis del sitio $\mathrm{CCH} 4$ (Parque Nacional Monte León, Santa Cruz, Argentina) e implicaciones para la arqueología de Patagonia. Magallania, 40(2), 221-231. http://dx.doi.org/10.4067/S071822442012000200010 .
Lobbia, P. (2016). Análisis morfométrico en Mytilus edulis: una herramienta para el análisis de los sitios concheros y la arqueología de la Costa Patagónica. Arqueología, 22, 145-161. https://doi.org/10.34096/arqueologia. t22.n0.3281

López, D. A., López, B. A., Arriagada, S. E., Mora, O. A., Bedecarratz, P. C., Pineda, M. O., González, M. J., Andrade, L. J., Uribe, J. M., \& Riquelme, V. A. (2012). Diversification of Chilean aquaculture: the case of the giant barnacle Austromegabalanus psittacus (Molina, 1782). Latin American Journal of Aquatic Research, 40(3), 596-607. https://doi.org/103856/vol40issue3-fulltext-9

Orquera, L. A., \& Piana, E. L. (2001). Composición de conchales de la costa del Canal Beagle (Tierra del Fuego). Segunda parte. Relaciones de la Sociedad Argentina de Antropología, XXVI, 345-368.

Pappalardo, P., Pitombo, F. B., Haye, P. A., \& Wares, J. P. (2016). A rose by any other name: systematics and diversity in the chilean giant barnacle Austromegabalanus Psittacus (MOLINA, 1782) (CIRRIPEDIA). Journal of Crustacean Biology, 36(2), 180-188. https://doi. org/10.1163/1937240X-00002403

Pastorino, G. (2000). Asociaciones de moluscos de las terrazas marinas cuaternarias de Río Negro y Chubut. Ameghiniana 37(2), 131-156.

Penchaszadeh, P. (1980). Ecología larvaria y reclutamiento del mejillón del Atlántico suroccidental, Mytilus platensis D'orbigny. Cahiers de Biologie Marine, XXI, 169179. https://doi.org/10.21411/CBM.A.5D0D670E

Piana, E., \& Estévez Escalera, J. (1995). Confección y significación de las industrias ósea y malacológica en Túnel VII. En J. Estévez Escalera y Vila Mitjà, A. (coords.), Encuentros en los conchales fueguinos, Volumen 1 de Treballs d'Etnoarqueología (pp. 239260), Bellaterra: CSIC Press.

Reimer, P., Bard, E., Bayliss, A., Beck, J., Blackwell, P., Ramsey, C., Buck, C.E., Cheng, H., Edwards, R. L., Friedrich, M., Grootes, P. M., Guilderson, T. P., Haflidason, H., Hajdas, I., Hatté, C., Heaton, T. J., Hoffmann, D.L., Hogg, A. G., Hughen, C. A.,... \& Van der Plicht, J. (2013). IntCal13 and Marine13 Radiocarbon Age Calibration Curves 0-50,000 Years cal BP. Radiocarbon, 55(4), 1869-1887.https://doi. org/10.2458/azu_js_rc.55.16947

Santiago, F., Gordillo, S., \& Salemme, M. (2014). Moluscos en contextos arqueológicos de la costa atlántica de Tierra del Fuego: Consumo Prehistórico e Implicancias de su distribución actual. Revista Chilena de Antropología, 
29 (1), 40-48. https://doi.org/10.5354/07191472.2015 .36204

Savoya, V. (2012). Mytilus spp. de Ambientes costeros del Atlántico Sudoccidental: ¿Nativas, exóticas o ambas? Córdoba, Argentina. Tesis Doctoral. Universidad Nacional de Córdoba.

Savoya, V., Gómez Otero, J., \& Schwindt, E. (2015). Toward a better understanding of the native-nonnative status of Mytilus mussels in the southwestern Atlantic: comparing pre-european middens and modern populations. Journal of Coastal Research, 31(3), 742-748. https:// doi.org/10.2112/JCOASTRES-D-13-00044.1

Scartascini, F. L. (2014). Arqueología de la pesca en la costa rionegrina, Patagonia Argentina. [Tesis Doctoral inédita]. Facultad de Filosofía y Letras. Universidad de Buenos Aires.

Seed, R. (1980). Shell growth and form in the Bivalvia. En D. C. Rhoads, y Lutz, R. A. (Eds.), Skeletal growth of aquatic organisms (pp. 23-67). New York, US.: Plenum Press.

Servicio de Hidrografía Naval. (2017). Tablas de Marea. Servicio de Hidrografía Naval, Armada Argentina. Recuperado de: http://www.hidro.gov.ar/oceanografia/Tmareas/ Form_Tmareas.asp

Steffan, P., \& Morsan, E. (2015). Un abordaje metodológico para el estudio de estacionalidad de captura de Mytilus platensis (d'Orbigny, 1842) en la costa norte del Golfo San Matías. En H. Hammond, y Zubimendi, M. A. (Eds.), Arqueomalacología: Abordajes Metodológicos y Casos de Estudio en el Cono Sur (pp. 105- 118). Buenos Aires: Fundación Félix de Azara.

Zaixso, H. E. (2004). Bancos de cholga Aulacomya atra atra (Molina) (Bivalvia: Mytilidae) del golfo San José (Chubut, Argentina): Diversidad y relaciones con facies afines. Revista de Biología Marina y Oceanografía, 39, 61-78. https://doi.org/10.4067/S0718 19572004000200003

Zaixso, H. E., Ré, M. E., \& Morsan, E. (2015). Moluscos costeros de interés económico, actual o potencial. En H. E. Zaixso y Boraso, A. L. (Eds.), La zona costera patagónica argentina: comunidades biológicas y geología (pp. 119-299). Comodoro Rivadavia: Editorial Universitaria de la Patagonia -EDUPA. Versión digital. Recuperado de: https://www.researchgate. net/publication/305816692_Moluscos_costeros_de_ interes_economico_actual_o_potencial [accessed Sep 17 2018]

Zubimendi, M. A., Castro, A. S., \& Moreno, J. E. (2005). El consumo de moluscos en la Costa Norte de Santa Cruz. Intersecciones en Antropología, 6, 121-137. 